\title{
Notes sur le phénomène érotique
}

Michel Henry

\section{Notes préparatoires à Philosophie et phénoménologie du corps et à L'essence de la manifestation.}

\section{Ms A 1-17-662 2}

Subjectivité du corps.

Il faut affirmer cette subjectivité (lire Merleau-Ponty), son importance pour la «perception» d'autrui (le corps de l'autre dont j'ai l'expérience dans l'amour sexuel par exemple, n'est pas du tout un objet, son mode d'être n'est pas du tout être-pour-autrui, i.e. quelque chose de transcendant, le sein que je caresse, c'est ce qu'il est subjectivement pour ma maitresse, c'est un acte transcendantal que j'appréhende. $)^{3}$

D'où le monde de l'amour : radicalement autre; la main qui serre la mienne et que je serre - c'est l'acte de serrer que je serre.

D'où le concept ambigu de Husserl de «corps organique » : ce n'est pas un objet mais un acte transcendantal d'autrui.

D'où la résurrection du corps. Il faut poser une nouvelle catégorie : «ce qui est susceptible de ressusciter», et «l'esprit impersonnel» en est tout aussi incapable que le corps-objet (qui n'est pas le vrai corps, le corps pour soi). ${ }^{4}$

(Il n'y a pas de «rapport» de ma conscience à la conscience transcendante si celle-ci est une hypothèse, un "être » inconscient**, et toute la philosophie de la conscience s'écroule et sombre dans le réalisme le plus vulgaire) (mais une conscience est transcendantale).

Le corps désigne seulement une certaine région des actes transcendantaux.

Le problème du corps peut être posé par une discussion sur le Traité des passions, corps (objet troublant l'esprit***) / Les Méditations (sentir c'est encore penser**** $\rightarrow$ corps : actes transcendantaux, conception opposée à celle de Kant (et Alquié)).

Rattacher au thème de la subjectivité du corps et du « tout est conscience » ma critique de la distinction de Scheler : esprit-vie. ${ }^{5}$

Subjectivité du corps : et pourtant, dans la mesure où je me représente ce corps objectivement, je le construis à partir de mon corps comme acte subjectif; donc il ne peut y avoir de faille absolue entre les deux (cf. moi empirique et transcendantal). Mais***** c'est cette construction objective de mon corps qui explique tous les arguments réalistes en faveur du dualisme traditionnel âme-corps (influence du corps sur l'âme, etc.)

*[Accolade pour tout ce paragraphe :] Sur [1']esprit. 


\footnotetext{
**[En note, au crayon :] On ferait du transcendantal un transcendant.

$* * *[$ En note, au crayon :] Ici corps = réalité sui generis « troublant» 1'esprit donc différent de lui.

$* * * *$ [En note, au crayon :] Ici cette réalité n'est plus opaque, hétérogène, elle est une intentionnalité, elle est esprit.

$* * * * *$ [Accolade au crayon pour le reste de la phrase :] Oui, le dualisme est une théorie transcendante.
}

\section{Ms A 15-2832}

Contre [le] naturalisme.

Si des désirs mauvais, de la concupiscence, on fait des penchants innés, on [perd] l'ambiguïté où l'individu, succombant dans l'impuissance de l'angoisse, devient à la fois coupable et innocent.

\section{Ms A 15-2835}

\section{Incarnation.}

Naïveté de Sartre : plusieurs intentionnalités [sont] possibles dans l'amour, bien différentes — [par exemple la] pureté ; mais déjà : «Ah, comme le buveur alors de l'acte étrangement s'évade ». ${ }^{6}$ (Le corps est l'œil de Dieu). *

$\neq$ Rousseau, Biran, naturalisme dualiste. C'est la faute à mon corps. Mais les corps seront jugés ; Rimbaud. ${ }^{7}[\ldots]$

*[En marge, dans une autre couleur :] Destin dans lequel ce qui faisait l'intériorité de sa vie et la fraîcheur de son expérience de vie sombre dans une objectivité fade et devient un élément mort dans la représentation.

\section{Ms A 15-2836}

Corps subjectif.

1/ La philosophie du corps subjectif rejette toute la philosophie hégélienne de la vie, i.e. aussi de la conscience de soi (dans le monisme). Car la vie est intériorisée, elle est la subjectivité elle-même, [non pas] quelque chose de transcendant, [une] région ontologique, par exemple [l'] objet de la biologie.

2/ Le fait que l'amour («physique») ne soit pas dégoûtant, [comme dans l']étonnement devant le « Da », l'être-là — [c'est parce qu']il est hors du monde, l'acte sexuel, et en un sens éternel, sacré et saint ; cf. l'erreur de Tropique du cancer, l'homme avec sa lampe de poche. ${ }^{8}$ Et c'est ce que Sartre connaît de l'amour, au point d'objectiver le sexe pendant l'acte sexuel ; je le manie, etc. ${ }^{9}$

Donc [il y a une] éthique de l'amour (physique), qui peut être pur ou impur, selon qu'il vise [le] « Da », ou au contraire la vie. 


\section{Ms A 15-2837}

Le corps n'est jamais nu (= en soi), mais toujours habité par ses gestes, ses intentions : même le corps objectif n'est pas objectif — $\neq$ appréhension de Husserl ${ }^{10}, \neq$ en soi, que voudrait bien devenir la liberté (pour-soi) de l'autre ${ }^{11}$-, mais c'est le corps subjectif apparaissant dans l'être transcendant.

$\rightarrow$ Le transcendant : pas [une] zone de l'être, mais du paraitre.

Donc le corps objectif apparaissant: [le] corps de l'autre n'est pas le corps du biologiste.*

*[En marge :] Oui ; problème que je ne traiterai pas, mais à signaler dans le dernier $\S .^{12}$

\section{Ms A 5-6-2842.}

\section{Autrui.}

L'acte sexuel est l'acte métaphysique non pas dans son sens, mais dans son essence.

Scheler : nous percevons même les mensonges d'autrui. Mais ce n'est pas aller si loin que cela [en] a l'air dans le psychisme d'autrui. Car le mensonge est l'acte réfléchi, pas spontané - il est donc décelable d'après un contexte, etc.; il y aurait plus à sentir ce que sent l'autre $\rightarrow$ acte sexuel.

\section{Ms A 5-6-2846/2847.}

\section{Autrui*}

L'alter ego n'est pas perçu. Le corps d'autrui n'est pas un objet innerweltlich. Le corps dans l'expérience d'autrui, le corps d'autrui est constitué comme un corps subjectif.

Sous la main de l'amant, le sein de la femme n'est nullement un être-là dans l'élément de l'être et de la généralité ; il est quelque chose qui se gonfle (cf. Pas d'orchidée pour miss Blandish ${ }^{13}$ ) (et ceci détruit toutes les descriptions que Sartre donne de l'autre). Dès que cela n'existe pas l'érotisme cesse** (une femme frigide)***. Donc c'est un corps subjectif. Problème : faire [l']ontologie de cet objet subjectif. Quelle est la possibilité de cet objet subjectif? Difficulté : comment ce qui en soi n'est pas constitué principiellement [pourrait-il] devenir accessible à travers un acte de constitution comme constitué ?

L'autre est objet perçu mais comme simple indice (cf. Sartre : imaginaire ; Husserl : apprésentation ${ }^{14}$ ). Fil conducteur : connaissance d'une intentionnalité dans le transcendant, méthode phénoménologique, Ego transcendant.

Mais cela, c'est comprendre :

1/ Notre attitude à l'égard de l'autre est-elle une attitude théorique, se laisse-t-elle réduire à une compréhension, haine, ressentiment, etc. ? 
2/ L'amour ne vise-t-il pas plus : la compréhension ne maintient-elle pas séparation: distance phénoménologique ? Toutefois distinguer problèmes existentiels et ontologiques.

*[En marge de la note, au crayon :] Sexualité.

** [En note :] Certes : cas de perversion, viol d'une femme étranglée, angoisse devant le Da.

***[En note :] L'érotisme, la corporéité comme élément objectif disparaît complètement. C'est cela la pureté. De même sourire, regard ; et si la jouissance est si importante (contre ce que dit Sartre ${ }^{15}$ ), c'est uniquement parce qu'en elle il semble que le subjectif soit finalement objet, que cette liberté en acte soit palpable tout d'un coup.

\section{Ms A 5-6-2850.}

\section{Sexualité.}

Contre Sartre.

1/ Comment peut-on s'emparer d'une liberté si on vise à la faire disparaître? Quand on dit qu'on ne peut la faire s'incarner pour la prendre (possession [dans $\left.1^{\prime}\right]$ amour ${ }^{16}$ ), façon de parler : on vise non le corps objet mais le « se donner », i.e. un acte intentionnel.

2/ Ce corps n'est pas la chose de Sartre. Ce corps des amants est une structure sexuelle, précisément il s'agit des sens et des fonctions.

Cf. La femme est pour moi une structure sexuelle offerte, ce qui peut s'offrir et ressentir.

On est aux antipodes de la chose [...]. C'est pour cela que la possession est quelque chose et qu'elle n'est pas le néant, c'est parce que ce sont deux libertés, deux Ego qui s'affrontent. Les corps des amants sont deux corps subjectifs (chacun est un corps subjectif non seulement pour soi mais pour l'autre, et réciproquement), donc deux subjectivités. Ce sont deux subjectivités qui font l'amour.

\section{Ms A 15-2852/2853}

\section{Expérience d'autrui, amour sexuel.*}

Une interprétation de l'amour sexuel est extrêmement difficile à donner à cause de notre liberté devant le monde; i.e. chaque subjectivité transcendantale donne à une même chose un sens différent, car ce sens est précisément ce que chacune est. Il y a [une] façon fasciste ou anti-fasciste de faire l'amour ; auto-érotisme ou fusion avec [le] cosmos. Cf. quand je m'allonge au fond d'une barque en mer et regarde le ciel : chute, abandon à [une] réalité impersonnelle ; j'ai l'impression d'être le mouvement même de la mer ou celui du passage même des nuages ; cf. Heidegger ; extase inférieure — dont je dois rendre compte — ou bien spectateur (désespoir) ; cf. dialectique de la réflexion: nous n'envisageons l'amour sexuel que pour autant qu'il s'insère dans le rapport à autrui, i.e. pour autant qu'il est amour (ou haine) ou volon- 
té quelconque d'atteindre autrui, d'appréhender autrui. C'est d'ailleurs ce qu'on cherche dans l'amour sexuel ; c'est pourquoi les amants, ceux qui s'aiment, se donnent l'un à l'autre, si purs soient-ils, si détachés de tout attachement pour une jouissance érotique quelconque. Ce que l'homme demande à la femme, ce n'est pas d'être objet, d'incarner sa liberté (et d'abord cela supposerait qu'il sache qu'elle est une liberté, cela supposerait l'expérience métaphysique d'autrui, $\neq$ Sartre). Ce qu'il veut, c'est appréhender l'acte de se donner, ce qui est un acte transcendantal de l'alter ego.

De même la femme [qui] veut être «prise », qui demande à l'homme d'être fort, de la prendre ; mais qu'est cette force : la force des bras qui l'étreignent? Nullement, mais l'acte transcendantal de l'amant qui prend. Amour, actes spirituels, projets (et si la femme fille a honte, ce n'est pas de son corps, mais c'est du pouvoir que l'autre a).

*[En marge :] Nous choisissons tout d'abord, pour défendre notre thèse d'une expérience métaphysique d'autrui, l'exemple le plus défavorable, celui de l'amour sexuel.

**[En note :] D'ailleurs, la femme ne se donne qu'après avoir éprouvé cette force comme acte transcendantal ; donc cette force n'est pas celle des bras, la force physique n'est qu'un symbole. Pourquoi l'homme veut-il prendre ? Pourquoi la femme veut-elle être prise? C'est le problème des sexes (cf. Sartre).

\section{Ms A 15-2855}

\section{Corps subjectif.}

Embrasser, c'est là quelque chose d'originairement invisible.

Cela est aussi un mouvement objectif*, et cela devient aussi objet d'une réflexion.**

Analyser : j'embrasse ma femme. On devrait dire ([s'il s'agissait d'un] « acte matériel»), mon corps embrasse, ou touche celui d'une femme - mais on dit: $J$ 'embrasse.

Alors, ou bien le caractère d'appartenance à l'ego d'un tel acte est figuré, symbolique ; mon moi se répand dans mon corps ; cf. psychologie, ses difficultés quand elle veut définir les limites du moi : cela serait moi d'une façon symbolique, comme ma cravate ou ma maison, comme ma main figure mon action dans le monde ; ou [bien] nous donnons à cette proposition un sens absolu, et alors corps subjectif $\rightarrow$ sérieux de l'existence. Nos actes ne sont pas regardés par une conscience transcendantale, [un] spectateur impartial***. C'est elle qui agit, embrasse, mange (cf. le Dasein mange-t-il ?)

*[En marge :] Et ce « aussi » pose évidemment le problème même de la métaphysique : pourquoi « deux ordres de faits », comme dit Biran ? ${ }^{17}$ Quant au rapport de ces deux ordres de faits, il doit être résolu à la lumière de la thèse kantienne revue par Lachièze sur les rapports des deux moi. ${ }^{18}$ 
**[En note :] Ha ! comme le buveur alors de l'acte étrangement s'évade. ${ }^{19}$ [À la suite, au crayon :] Qu'on ne prenne pas prétexte de cela pour dire que le corps est objectif : le destin qui menace tous les actes du corps menace aussi tout acte spirituel.

***[En marge, au crayon :] qui pourrait toujours les désavouer même au moment où elle les exécute; certes cela est possible, mais c'est une modification qui vaut aussi bien pour n'importe quel acte spirituel.

\section{Ms A 15-2860}

\section{Corps.}

Montrer que le principe d'une description érotique doit être subjectif (cf. ce que je ferai dans La faim $^{20}$ ). Pourtant, les descriptions pornographiques qui sont objectives (ce qui montre leur erreur) sont [des] suggestions : parce qu'elles relèvent du Da (qui est une intentionnalité érotique détournée : le mal. Or c'est nécessairement cette forme que prend l'auto-érotisme). [...]

Il faut faire une philosophie idéaliste du corps et (contre Merleau-Ponty), il faut donner à la philosophie idéaliste du corps un développement radical.

\section{Ms A 15-2861}

\section{Corps. *}

Réhabiliter le corps grâce au corps subjectif.

Bien montrer que le corps n'est pas condamné par l'Église par exemple, mais [que] le «corps » comme le «monde » est un concept existentiel, désigne une certaine intentionnalité, celle du « Da », du regard sur $\operatorname{soi}^{21}$ : se toucher ; quelque chose apparaît dans le transcendant et j'y cherche un mystère car c'est de nouveau l'histoire du tombeau vide, du passé, etc. ${ }^{22}$

Voilà l'échec de l'amour physique, lorsqu'il obéit à cette intentionnalité-là.

Le problème du pour-soi qui veut se faire en-soi est très mal formulé ; le désir de trouver, c'est cela l'illusion du corps, celle de la dialectique de l'objectivation ; mais esthétique religieuse : intentionnalite..$^{23}$

*[En marge, au crayon :] « Corps chrétien ».

\section{Ms A 5-6-2865/2866.}

Sexualité (corps conclusion)*24

Dans la chair (corps-objet) de l'autre : ce qui est absolu semble contingent; il peut être là plutôt qu'ailleurs; il pourrait, semble-t-il, ne pas être là. Mais voilà l'important : ce n'est pas seulement sous cette face contingente que l'absolu semble accessible (amour : désir sexuel du corps de l'autre). Mais illusion, échec de cette voie : ce qui semble accessible seulement de cette façon est bien plutôt inaccessible $\rightarrow$ répétitions de la vanité et de l'échec car le plaisir (la jouissance) est quelque chose de transcendant, je ne puis m'y perdre, j'en suis toujours séparé (en cela 
Sartre a raison $\left.{ }^{25}\right)^{*}$. C'est seulement dans ce dont je ne suis pas séparé que le salut peut résider: y a-t-il un tel élément? Oui, l'intentionnalité. C'est plutôt dans le caractère sacré du geste que la solution est à chercher. Et il faut encore faire ici cette remarque : cette intentionnalité (ce geste) ne serait pas ce qu'il est s'il n'était geste vers, avec l'autre. Donc pas question ici de solitude subjective; bien plutôt c'est dans et par la tonalité affective de l'Erlebnis considéré, donc dans ce que devient ici l'essence de la subjectivité en tant que telle que la solitude est levée. Ainsi est résolu à la fois sur le plan ontologique et sur le plan moral le problème de l'expérience d'autrui.

*[En note, au crayon :] TB.

\section{Ms A 28-5641}

Max Scheler et les amants. *

Il reconnaît l'importance très grande de l'amour sexuel (contre « la vieille morale des prêtres ») mais il lui refuse aussitôt, au fond, toute signification pour la connaissance d'autrui, puisqu'il ne voit dans l'amour sexuel qu'une absorption de l'individu dans des forces cosmiques et vitales impersonnelles et universelles. Alors l'autre n'est qu'un prétexte à cette identification cosmique et n'importe quel « autre » devrait pouvoir remplir ce rôle.

Cette erreur et ses conséquences découlent de sa distinction conscience-vie et de la position d'une sphère autonome de la vie non-conscientielle, ce qui n'a aucun sens car même une fusion cosmique est consciente, éprouvée dans le cogito concret. ${ }^{26}$

*[En marge, dans une autre couleur :] Autrui.

\section{Ms A 28-5642}

Amour-amants. *

Double exigence, contradictoire : désir de fusion, de se perdre dans l'autre ; désir d'être tout pour l'autre, d'être l'essence. Élucider ceci.

Mais on ne peut naturellement [pas] partir d'une description de ce genre pour saisir l'expérience d'autrui, car la description de ces deux mouvements suppose évidemment l'expérience d'autrui, donc l'autre avec lequel s'institue cette dialectique.

*[En marge :] Autrui-amour.

\section{Ms A 28-5644}

Cf. L'être et le néant, p. $649{ }^{27}$

Cf. cristallisation stendhalienne.

Si l'amour vise à s'emparer du monde entier, il ne vise pas à s'en emparer à travers un corps, celui de telle femme (« qui ne représente qu'un corps conducteur qui est placé dans le circuit »), mais il vise un religieux et à travers lui le monde ou un monde, dont le religieux est la condition (et dont « un monde » est le monde). Mais parce que le religieux qui vise le religieux veut se l'approprier, il le manque. 
Étudier les rapports de l'avoir et de l'esthétisation.

\section{Ms A 28-5646}

Dieu est mort.*

C'est la « dure parole » de la conscience malheureuse, mais celle-ci est une conscience sensible, est une conscience d'erreur - et d'ailleurs tout ce qui est dur est faux.

Quant à Nietzsche, il a dit qu'il fallait tuer Dieu; proposition contradictoire, comme tout ce qui relève du ressentiment. De plus c'est là, semble-t-il, une entreprise difficile; il est vrai qu'avec les progrès de la science et de l'énergie atomique...

Contre Sartre : on ne peut évidemment parler de Dieu à l'intérieur de la dialectique Maître-Esclave, puisqu'on en parlerait à l'intérieur du ressentiment — sphère [avec laquelle] il n'a rien à voir. ${ }^{28}$

*[En marge, au crayon :] Ressentiment.

\section{Ms A 28-5647}

\section{L'amour.}

Il n'y a d'expérience d'autrui que pour celui qui aime.

- Opposer amour à exécration, i.e. ma conception de la subjectivité transcendantale [à] celle de Heidegger. Mais l'exécration existe ; seulement, ce que Heidegger donne comme la structure fondamentale de la conscience n'est qu'un mode possible qu'elle peut se donner parmi d'autres — précisément, la haine, ou le nihilisme.

- Établir [la] solidarité haine-nihilisme (destruction). C'est seulement lorsque le sujet n'est rien qu'il peut être ce qui n'est pas l'objet, ce qui n'est que négation (haine) ; dans la négation qu'il porte, le sujet de Sartre est essentiellement une réaction. Cf. Nietzsche. C'est une philosophie d'esclave, de pauvreté. L'ironie, qui est le mode le plus aimable de l'exécration, est encore une forme d'exécration (cf. Giraudoux) (pas celle de Socrate, qui est possession du sujet, recueillement religieux).

N'est-ce pas cela : aimer? Eprouver la liberté d'autrui, en avoir une expérience directe et concrète, et non chercher à l'annihiler, comme le pense Sartre ?* Et même dans l'amour sexuel (et non [1']amour "physique », [1']instinct sexuel, [qui relève] du naturalisme incapable de comprendre quoi que ce soit) : le sein d'une maîtresse n'est pas un objet; ce qui veut dire Rilke lorsqu'il dit des amants : «Vous qui sous les mains l'un de l'autre devenez abondants comme des années de raisin $»^{29}$; c'est la vie intérieure de l'autre que j'atteins (comme subjectivité transcendantale).

(Dira-t-on que c'est là situer l'esprit? Nullement; il n'est situé que comme moi empirique, mais précisément l'expérience d'autrui est métaphysique).

La conception de Sartre de l'amour sexuel repose sur un contresens absolu, sur l'idée du corps qu'il croit être [un] objet, d'où son effort pour inclure la liberté de l'autre dans l'objet (ce qui est assurément une tâche impossible, aussi impossible 
que le fantastique projet d'inclure le pour-soi dans l'en-soi); on oublie que ce n'est nullement d'objets que les amants ont l'expérience, bien que cette réalité dont ils ont l'expérience leur apparaisse aussi comme objets dans le monde de la représentation, tout de même que mon moi m'apparait aussi comme moi empirique. ${ }^{30}$

*[En marge :] D'ailleurs, comment pourrait-on chercher à l'annihiler, si l'on n'en avait pas cette expérience concrète?

\section{Ms A 28-5649}

\section{L'amour.}

Même l'amour «physique » (sexuel) est une expérience métaphysique.

C'est l'autre qui est atteint, i.e. l'autre comme acte de se donner, comme m'aimant, i.e. comme acte transcendantal d'un corps concret dans sa détermination religieuse la plus haute (d'où le caractère sacré de l'amour sexuel, reconnu par l'Église qui fait du mariage un sacrement).

Cf. Rilke: «Vous qui sous les mains l'un de l'autre devenez abondants comme des années de raisin $» .^{31}$

Le processus et le telos de l'amour sexuel est donc le contraire de celui que décrit Sartre pour qui on vise dans l'amour à réduire l'autre, à la chosifier pour l'appréhender, ce qui est certes impossible et qui est de plus le contraire de l'amour. ${ }^{32}$

Mais la vanité du projet amoureux — selon lui — n'empêchera pas les gens d'aimer. Cette impuissance de sa philosophie sur ce point en est une condamnation. La philosophie doit aller dans le sens de la nature, la rendre intelligible.

Mais il peut y avoir acte sexuel sans qu'il y ait amour sexuel ; cela ne signifie pas du tout qu'ici l'amour serait bien physique; mais simplement que le projet qui commande un acte n'est plus l'amour mais le désir - encore celui-ci peut-il avoir bien des sens : auto-érotisme (c'est à ce stade seulement que s'appliqueraient les descriptions de Freud) ; sadisme, masochisme, etc., comme on le croit. Et c'est ici la faillite de toute la dialectique hégélienne par exemple: une même détermination objective (dans l'être transcendant) peut avoir divers sens, il peut être vécu transcendantalement de bien des façons différentes*. ${ }^{33}$

Par conséquent, faire une analytique de l'amour sexuel par exemple est vide de sens ; car elle ne saurait avoir une portée générale, à moins qu'on entende « amour » au sens strict; mais une analytique de l'acte sexuel, elle, n'a aucun sens, car quelle est mon attente à moi qui accomplit cet acte, qu'elle est le sens que je lui confère (amour, auto-érotisme, etc.) ? C'est ici la ruine de l'analyse de Sartre, qui ne donne pas une analyse de l'amour sexuel, mais qui élève à l'absolu une expérience particulière (dont je dois trouver le nom et qui est la sienne - auto-érotisme sans doute.)

*[En note :] Et c'est aussi la ruine de l'analyse que Heidegger donne du concept de monde, car le monde peut être vécu transcendantalement de façons bien diffé- 
rentes : le rapport à un monde fini ne saurait enchaîner la subjectivité et lui donner une vie et un sens finis.

\section{Ms A 28-5651}

Amour.

Contresens énorme de Sartre sur l'amour, qu'il décrit à l'intérieur de la dialectique Maître-Esclave, alors que précisément l'amour est ce qui y échappe.

C'est pour cette raison que Dieu n'a pu combattre Lucifer (ceci a été aperçu par Boehme, p 158: «comment l'aurait-il fait, dit Koyré, en s'embrasant lui-même et en dardant sur Lucifer les rayons ardents et fulgurant de sa colère ?» $\rightarrow$ Mais Dieu non seulement aurait « annihilé et réduit en cendres la nature », mais se serait détruit lui-même). ${ }^{34}$

C'est pour cela que le Christ dit : aimez vos ennemis, pour ne pas tomber dans la dialectique Maitre-Esclave ; mot qui prend ainsi une profondeur inouïe - et on [comprend] la thèse de Nietzsche : haïr, c'est être esclave. ${ }^{35}$

\section{Ms A 28-5652}

Amour-mort.

Il y a une grande ressemblance entre la fin d'un amour et la mort que quelqu'un sent venir.

Ainsi quand la mort vient — les projets : agrandir la maison, aménager le jardin, et puis retourner à tel endroit, tout cela se profile tout d'un coup avec un fond tragique de rien, comme n'étant plus soutenu par rien; et d'où cela vient-il, sinon du fait qu'il n'y aura pas de subjectivité, donc [qu']un monde sans vie va s'écrouler?

De même, fin d'un amour : aller se promener, faire telle ou telle chose; tout cela qui semblait des occupations extérieures perd tout fondement, se révèle vain, sans attrait, et même sans existence, [et] révèle quel est vraiment son être, maintenant que cet être n'est plus : cet être, qui semblait extérieur, c'était en réalité la subjectivité, l'intentionnalité, et l'amour.

\section{Ms A 28-5653}

Amour.

Sartre incapable de distinguer orgueil (vouloir être reconnu) et amour.

\section{Ms A 28-5655}

Faire une psychanalyse de l'amour analogue à ma psychanalyse du père.

Dans l'amour, je me confie à l'autre, mon être lui est remis, mais il faut qu'il le soutienne, i.e. qu'il m'aime; mon être est par son amour, j'abandonne mon être à l'acte de sa liberté, mais ainsi il est vital pour moi que cet acte par lequel il fonde mon être en m'aimant ne s'arrête pas; d'où ma jalousie, d'où besoin de présence, pas tant de présence physique que de l'annonce que cet acte de sa liberté qui fonde 
mon être se répète et ne cesse. Embrasser, vouloir embrasser, c'est cela. J'embrasse pour qu'on m'embrasse et voilà pourquoi on s'embrasse sur la bouche. L'acte par lequel on m'embrasse, c'est pour moi l'acte par lequel mon être est posé, l'acte par lequel on m'aime.

Donc le mouvement des lèvres de la femme qui s'avance vers moi, si elle va m'embrasser, n'est pas perçu comme un mouvement objectif mais comme ce qui fonde mon être ; et si elle s'arrête dans son mouvement, mon visage change parce que mon être est en question : inquiétude de l'amant : qu'est-ce qu'il y a ? Quel rapport avec le geste lui-même ou même avec le plaisir sensuel ? C'est de tout autre chose qu'il s'agit.

Fin [de ma] thèse : nous ne devons pas chercher à ce que les autres nous aiment. Nous aimer, c'est là leur tâche à eux, et ce n'est nullement notre affaire. Mais c'est une tâche à laquelle nous n'avons même pas le droit de les introduire. Si la morale devait ici prendre la parole, elle dirait sans doute : veuille que les autres aiment, mais ne cherche pas à ce qu'ils t'aiment.

\section{Ms A 31-6198}

Sexuel.

Le critère du sexuel doit être le corps subjectif, ou plutôt l'intentionnalité. Les zones érogènes ne sont que des pôles d'un monde érogène qui correspond à une certaine intentionnalité.

\section{Ms A 31-6200}

Sexualité.

Quelle est l'intentionnalité qui procède à la sexualité - i.e., que devient le « corps dans la sexualité »?

Question à laquelle répond Sartre comme si une seule intentionnalité pouvait présider à un acte matériel - ce que je nie.

C'est seulement le corps objectif qui fait toujours la même chose, ces pauvres gestes, toujours les mêmes, sur lui seulement on pourrait s'apitoyer. Moi : le corps subjectif - le sérieux.

\section{Ms A 31-6201}

Le sexe est un être-là mystérieux. Cf. Miller. ${ }^{36}$

Expliquer pourquoi.

Ms A 31-6202

Que l'acte sexuel ne s'épuise nullement en sensations (auto-érotisme), c'est ce que montre :

1/ Le corps subjectif (il est certes subjectif avant d'être sensations); 
2/ L'intentionnalité vers l'autre, mise également en lumière dans les cas de perversions solitaires. L'insuffisance de l'acte solitaire se manifeste dans les fantasmes qui l'accompagnent, et même le plus souvent le sujet se voit dans un rapport avec l'autre (le plus souvent un rapport passif).

\section{Ms A 31-6203}

Le pansexualisme de Freud.

[Il] ne peut nullement signifier l'impérialisme d'un besoin (sexuel) sur les autres ; car il y en a d'autres aussi impérieux — par exemple le besoin de manger.

Donc si le pansexualisme est possible, c'est en se niant précisément en tant que doctrine « biologique ».

D'ailleurs, dissociation entre exigence sexuelle et exigence physiologique des organes sexuels, visible par exemple dans la jalousie.*

Donc la sexualité a une signification ontologique, « projet»; Sartre a raison. Mais maintenant, quel est son sens ? Sartre a-t-il décrit convenablement ce projet ? [...]

\section{Ms A 31-6204}

L'activité sexuelle intense de certains individus, hommes ou femme, ne s'explique pas physiologiquement, elle se comprend; elle est une psychose.

Dire en quoi celle-ci consiste : faire la preuve de l'amour, même seul, ou trouver le secret (cf. Kierkegaard) du mal ; ou angoisse devant le « Da »; ou Freud, sensations agréables qu'on veut se redonner - mais ce caractère « agréable », jouissance, est en fait un sens (sens de la jouissance, voir Hegel, Sartre) qu'il faut élucider, et qui ne peut se comprendre que dans le contexte de toute l'expérience humaine.

\section{Ms A 31-6205}

\section{Sexualité.}

Réfutation de la thèse naturaliste du freudisme, dans la mesure où il y est lié ; la sexualité serait un besoin naturel, cf. manger, et qui demanderait à être satisfait comme lui. Si on ne le satisfait pas $\rightarrow$ refoulement et perturbation graves.

Malheureusement, cette thèse s'écroule devant les faits [...]; la sexualité n'est rien de «naturel», elle a un sens ; [pour] preuve ses variations, [dans la] jalousie, etc. «Faire l'amour » n'est pas à analyser comme manger, c'est «faire la preuve de l'amour ".

Donc utiliser la sexualité jalouse (Lagache) ${ }^{37}$ pour avancer la thèse : amoursexuel-intentionnalité, et montrer sur ce point la contradiction où s'enferme le freudisme : naturalisme rationnel.

\section{Ms A 31-6206}

Amour sexuel.

Absurde : « contact de deux épidermes », etc. 
Les amants n'ont pas conscience de leurs organes comme organes, i.e. [comme de] structures physiologiques, ils font l'amour* ; "c'est comme la mer » - et la mer non comme objet de comparaison, mais comme mouvements** — i.e. subjectif.

*[En marge :] Le sensible n'est pas sensible (il est une intentionnalité).

**[En marge :] Voir Bergson, problème du mouvement.

\section{Notes préparatoires à C'est moi la vérité et à Incarnation}

\section{Ms A 32-24-24482}

Parler d'acte naturel, c'est parler d'un cercle carré ; car il n'y a pas et il n'y a jamais eu dans la «nature» un seul «acte». Aussi est-il absurde de parler de la sexualité comme d'un phénomène naturel.

Pour le christianisme il n'y a aucune sexualité naturelle, mais seulement un amour sexuel, éventuellement la déchéance de cet amour au rang de ce qui est considéré par les partenaires comme naturel, mais qui ne l'est jamais.

C'est parce que la sexualité n'est pas naturelle qu'elle peut déchoir ou se métamorphoser en toutes les formes de la vie, sadisme, masochisme, et devenir un mal, une profanation ; [...] le rapport du monde moderne à la sexualité est essentiellement un rapport de profanation ; traiter la sexualité hors [de la] vie et ses prescriptions internes, ce n'est pas seulement [une] ruine morale, mais [c'est aussi un] nonsens intellectuel. ${ }^{38}$

\section{Ms A 32-24-24485}

Autrui. Sexualitélérotisme. Naturel. Sexualité [conçue comme] quelque chose de naturel.

Alors qu'on dit aujourd'hui que la sexualité est quelque chose de naturel: en fait, dans l'érotisme, on veut toucher le plaisir, le désir, l'impression de l'autre, son âme et - comme on ne les touche pas et qu'on ne le sait pas - on regarde dans ses yeux pour voir si l'on y parvient.

C'est un moyen de pénétrer dans la vie de l'autre dans le monde; et c'est, réduit à cela, un échec total ; il n'y a d'érotisme que dans l'amour sexuel, [la] fusion amoureuse, i.e. ailleurs, dans la vie.

Dans le monde d'aujourd'hui, dans la société actuelle, la sexualité est le seul reste de vie spirituelle.

\section{Ms A 32-29-24589}

$[\ldots]$

Vie selon [la] chair $\rightarrow$ aspirations charnelles ;

Vie selon [1']Esprit $\rightarrow$ (aspirations) spirituelles.

Donc si [la] chair $=$ [un] mode de vie, il s'agit d'un mode de vie qui aspire à [la] mort; le décrire : finitude-chose-sexe, ressentiment, haine [de la] vie. 


\section{Ms A 32-29-24590}

Romains 12 : nous ne sommes pas tenus vis-à-vis de la chair de vivre selon la chair (= mourir).

Ms A 33-10-24702

Amour (Vie/vie).

L'amour est la conscience de Dieu en nous ; cette conscience naît souvent à la faveur du prochain lorsque l'autre est perçu en lui-même, i.e. en Dieu.

\section{Ms A 35-01-25888}

Désespoir dans l'érotisme?

Paganisme - faire passer en quelque sorte «l'intérieur » de la peau à l'extérieur ; là où le soleil chauffe, là aussi, semble-t-il, est le plaisir ; le plaisir de la nudité [distinct] de l'innocence qui ne sait pas qu'elle est nue; elle ne connaît pas l'angoisse du paradoxe kierkegaardien. ${ }^{39}$

[À la suite, dans une autre couleur :] En ce sens, se débarrasser de soi en le rendant public [...]. Y a-t-il un désespoir caché sous l'érotisme ? Sur un plan général, $y$ a-t-il une tristesse du sensible ? (de la Grèce par exemple)?

\section{Ms A 35-01-25889}

Echec de l'érotisme (cf. thème «le menteur ») — signes simulés dans tous les cas :

- Simulation inhérente à [l']érotisme $\rightarrow$ voyeurisme - pas seulement le fait de celui qui voit mais de celui qui est vu, s'offre à la vue ; échangisme/voyeurisme ;

- Prostitution inhérente au voyeurisme ; [elle n'est] pas réservée à l'argent mais [au] fait même de se prostituer en public [...].* [Cette prostitution inhérente] à tout érotisme impliquant une venue dans le monde de l'extraordinaire, de la vie ; qu'il échoue $\rightarrow$ répétition indéfinie.

Profanation; cf. paganisme.

$*$ [En marge :] Exhibitionnisme. ${ }^{40}$

\section{Ms A 35-01-25900}

Le Menteur.

En quoi Satan est le menteur?

- Parce qu'il propose le pouvoir (du monde) quand la vérité est le non-pouvoir.*

- En quoi est-il le menteur sur le plan charnel (la séduction) (voir aussi la femme selon Kierkegaard, pas plus coupable).

*[En marge :] Il propose aussi d'atteindre, de prendre la réalité de l'autre, ce qui est impossible, même dans le cas de la bonne volonté de l'autre ; en ce sens la réalité n'appartient qu'à Dieu). 


\begin{abstract}
Ms A 35-01-25901
Le Menteur (le péché de la chair).

Le mensonge est de faire croire à l'homme qu'il pourrait avoir autre chose que ce qu'il a déjà, i.e. la Vie ; pour cela il convient de faire miroiter autre chose que ce qu'il a, que sa réalité, la possibilité qui n'est rien (montrer ensuite comment cette dialectique trouve son « incarnation », sa réalisation, dans la durée).

Le péché se présuppose lui-même mais il est la présupposition de l'irréalité ?
\end{abstract}

\title{
Ms A 35-01-25904
}

Érotisme : cette connexion chair / impression constituée — désir.

Désir: tentative de saisir la vie de l'autre sur son corps objectif, pratique d'incantation pour [provoquer l']incarnation de [sa] chair réelle [dans son] corps objectif ; sentir son plaisir sur sa peau, son sexe...

\section{Ms A 35-01-25907}

1/ Du touchant/Je peux immanent au touchant intentionnel (toucher dans l'extension, dans l'extérieur, ce qui n'y est jamais).

2/ Critique [de l'] onanisme.

Répétition, redoublement de l'échec dans le monde.

Cf. auto-érotisme, onanisme — rapport [de] soi à soi ; mais le soi se dérobe à ce rapport à lui-même dès qu'il est cherché dans le monde - échec [de l']autoérotisme. ${ }^{41}$

\section{Ms A 35-01-25922}

\section{Érotisme.}

Se donner n'est qu'un acte symbolique, s'il s'agit de donner non plus seulement son corps mais sa chair. Pourtant, ne donne-t-on pas aussi le plaisir ? On ne peut le donner que là où il se tient; où ? Dans la vie. Mais ne le partage-t-on pas ? Mais la vie ne se partage pas, il n'y en a qu'une ; c'est seulement là où se tient la vie qui ne se partage pas que l'union s'accomplit, mais pas dans le plaisir objectivé ; là elle se dérobe toujours.

\section{Ms A 35-01-25923}

Erotisme / sensualisme.

Ce qui est constamment occulté dans l'érotisme, [entendu comme] auto-érotisme - avec [l'appel aux] sensations des sens, [au] donné empirique, cf. Husserl, «alors... si...») — ce sont les tonalités affectives fondamentales liées à la vie comme telle et indépendantes des sensations corporelles (= ayant leur principe dans le corps chosique / matériel) — ici liées à [l']analyse [de la] passivité, création/génération. 


\section{Ms A 35-01-25924}

Auto-érotisme.

L'homme veut se changer.

Cf. Hitler, Staline, Nietzsche.

\section{Ms A 35-02-25933}

Péché de la chair, échec [de l']érotisme.

Contrairement à la Vie, la chair désire être vue. C'est pourquoi elle se dénude ; ce faisant elle se fait voir comme corps, mais comme un corps qui se sait chair, qui est chair, non pas toutefois dans sa monstration mondaine. ${ }^{42}$

Désir [de] séduction (Le menteur), le pouvoir.

$\rightarrow$ Ici est à rappeler à l'homme qu'il n'est pas le pouvoir et que le pouvoir de la chair de se montrer en elle-même, comme fille de ce pouvoir, lui est retiré.

D'où vient cette possibilité de l'opposition de la chair à la Vie ? De l'idolâtrie du Je peux, du toucher-moi, du [fait de] produire des sensations en moi.

[À la suite, dans une autre couleur :] Le Je peux (autonomisé) est donc bien la source de l'(auto)érotisme et du péché : double idolâtrie du je peux, de [la] chair ravalée [au rang d']objet $\rightarrow$ paganisme, profanation. ${ }^{43}$

\section{Ms A 35-03-25949}

Le péché de la chair.

À propos d'Irénée, p. 366 . $^{44}$

Pourquoi la chair est-elle le lieu par excellence du péché ? Parce qu'elle est celui du toucher double, le lieu où l'homme seul (ou aidé d'un des ses complices) peut faire naître en lui les sensations de plaisir qu'il veut; là où il se prend lui-même pour fin au sens du plaisir auto-érotique. Ainsi la chair est-elle le lieu où tout amour, tout désir peut se changer en le contraire de soi — en amour idolâtre, en auto-érotisme [À la suite, dans une autre couleur :] L'érotisme comme le contraire de l'amour.

[...] Parce que la chair porte en elle cette possibilité permanente de détourner sur soi l'amour du soi pour la vie et l'archi-vivant, elle est le lieu du péché, et rejoint l'acte de Lucifer : ne devoir rien à personne, à la Vie, mais avoir la possibilité de produire en quelque sorte la vie et sa propre vie et ce sous la forme d'un plaisir.

Que cette possibilité soit une illusion, tout le dit: ni [la] chair ni [la] vie ni même ce plaisir ne sont leurs propres fondements, mais dans la possibilité de pouvoir [réside celle de faire] advenir ce plaisir. Le Soi/moi accomplit concrètement le retournement de la passivité en activité ; je peux toucher et donc déterminer une sensation en moi ou en l'autre : « le faire jouir ».

La possibilité concrète du péché repose sur une semi-illusion : que l'homme est son propre fondement, qu'il ne doit rien à Dieu. 
Le dispositif divin de l'amour est détourné de sa finalité pour devenir celui du plaisir ; déjà en se déshabillant, en se découvrant soi-même comme chose, l'homme a l'illusion qu'il est une chose et qu'il a l'autonomie des choses.

Ainsi tout comme l'homme en général, la chair a l'illusion d'être autonome, donc libre $\rightarrow$ libération sexuelle... Tout cela devient l'objet d'une économie autonome et non plus divine. ${ }^{* 5}$

*[En marge, dans une autre couleur :] La religion remplacée par l'érotisme.

\section{Ms A 35-03-25952}

Le péché de la chair ; [à la suite, dans une autre couleur :] Transition avec salut. Le serpent est d'abord le Menteur. En quoi ?

À la place de l'innocence, il fait miroiter à travers la séduction le pouvoir de la chair (ego) sur elle-même, sur l'autre - le schéma de Condillac, cf. la tentation du Christ, le Christ prêche le non pouvoir de l'ego = Dieu, l'humilité qui selon tous les grands mystiques libère la place pour ce qui s'y trouve avant elle $=$ Dieu.

De plus ce pouvoir est une illusion, un pouvoir relatif, temporaire, et accordé, ce qui vient établir la mort.

\section{Ms A 35-03-25954}

Le je peux / trompeur.

Un trompeur extraordinaire en ce sens que ce qu'il dit est vrai.

1/ Il est vrai que je peux; et cependant ce n'est pas moi qui peux à proprement parler $; 1^{\text {ere }}$ tromperie $; 2^{\text {ème }}:$ je peux atteindre l'autre $=$ faux (je ne le peux que dans le non-pouvoir). [...]

[L']Ego suppose [1']ipséité et [le] pouvoir aussi $\rightarrow$ tu n'aurais aucun pouvoir s'il ne t'avait été donné d'en haut ;

2/ Chair, lieu de la réalité

L'auto-donation du pouvoir équivalente en certitude à celle de la douleur (cogitatio) mais de même que [l']impression [n'a de réalité que] dans la vie, de même [le] pouvoir.

\section{Ms A 35-03-25955}

Analyse de l'illusion transcendantale du « je peux » dans son rapport à [l']analyse du Je. « Je » aussi bien que " peux » sont à la fois réels et illusoires ; et $J e \rightarrow$ ipséité $\rightarrow$ condition du pouvoir.

Ms A 35-03-25958

Péché de la chair.

Je peux est affectif; orgueil — mon corps est à moi. 


\section{Ms A 35-07-26009}

Faire une phénoménologie de la convoitise de la chair.

Cf. chair vertigineuse. ${ }^{46}$

Impossible de saisir — Sehen und Fassen — l'autre [chair] (là où elle touche à elle-même avant de pouvoir toucher et être touchée) mais vertige de cette possibilité.

(On ne peut pas plus saisir l'autre que soi-même / soi-même que l'autre, en tant que Soi originaire).

\section{Ms A 35-07-26025}

Érotisme - sensible contre affectif, ambiguité du sensible.

L'avantage de l'érotisme est de nous placer immédiatement hors de la pensée.

Dira-t-on pour autant: dans la vie? C'est là ce que nous devrons contester. L'érotisme nous place plutôt dans le sensible, mais il faut pour entendre l'érotisme à partir du sensible et comme sensible tracer une ligne de démarcation que seule la phénoménologie de la vie a su tracer, entre le sensible et l'affectivité, entre le sensible et la Vie.

Car le sensible, s'il procède du sentir, nous a jetés d'emblée hors de la vie ; le sensible est donc en soi quelque chose de mort, et il n'est vivant que pour autant que ce qui est devant dans l'apparaître du monde vit aussi dans la vie — ne vit dans le même temps que dans la vie. L'érotisme nous apparaît alors comme la vérité, la tentative, la tentation de saisir la vie, de la voir, dans le monde.

Tel est le désir, tel est son échec.

\section{Ms A 35-07-26027}

Péché = Désir, non chair ou corps.

Romains 6: le péché est celui du désir; quel désir est le péché? Celui de l'idolâtrie ; il n'y a qu'un péché. ${ }^{47}$

Cf. Egoïsme transcendantal.

\section{Ms A 35-07-26029}

L'autre se dérobe au Je.

Il y a une expérience d'autrui parce qu'il y a un échec de l'expérience d'autrui, i.e. parce que ni ego ni alter ego ne sont autonomes, parce qu'il y a en chacun un en deçà de chacun...

[À la suite, dans autre couleur :] Cf. Kierkegaard, l'angoisse ; que moi ou toi soient là = toucher une autre incarnation, dans la visibilité ; se dénuder ne suffit pas ; se donner avec [de] multiples signes de jouissance. 


\section{Apparat critique}

${ }^{1}$ Nota bene : pour l'édition de ces notes, les ajouts entre crochets ([ ]) indiquent une intervention ou une indication de l'éditeur, les points de suspension entre crochets ([...]) une coupe de l'éditeur.

2 Les manuscrits A $1-17-662$, A 5-6-2842, A 5-6-2846/2847, A 5-6-2850, et A 5-62865/2866, furent initialement édités dans Michel Henry, «Textes inédits sur l'expérience d'autrui », Revue Internationale Michel Henry, $n^{\circ} 2$, Presses universitaires de Louvain, décembre 2010. En raison de l'unité thématique qu'ils forment avec ceux que nous publions ici, nous avons pris la décision de les y adjoindre.

${ }^{3}$ Sur la manière dont Henry aborde, à l'époque de la rédaction de ces notes, la question de l'expérience d'autrui en général, cf. Michel Henry, «Textes inédits sur l'expérience d'autrui », op. cit. ; pour une explicitation du concept d' "expérience métaphysique d'autrui », mobilisé ici à plusieurs reprises, voir aussi notre présentation, « De l'expérience métaphysique d'autrui à l'intersubjectivité en première personne », p. 16-70.

${ }^{4} \mathrm{Cf}$. sur ce point Philosophie et phénoménologie du corps. Essai sur l'ontologie biranienne, Paris, PUF, «Epiméthée », 1965, p. 289 : « Les Corinthiens s'en allèrent en ricanant lorsque saint Paul prétendit ne pas réserver à l'âme le privilège de cette résurrection. Il est clair, au contraire, que si l'être originaire de notre corps est quelque chose de subjectif, il tombe, au même titre que 1" "âme", sous la catégorie de ce qui est susceptible d'être répété et d'être jugé. »

${ }_{5}^{5}$ Ce que Scheler, dans Nature et formes de la sympathie, nomme «sphère vitale » est « la sphère qui comporte l'existence métaphysique d'une "vie" supra-individuelle dans tout ce qui est vivant, d'une entéléchie primitive dans tout être soumis à l'action régulatrice de forces vitales » (trad. fr. M. Lefebvre, Paris, Payot, 1928, p. 61) ; et c'est d'abord eu égard à cette sphère que valent les phénomènes de la sympathie, et que deviennent intelligibles, selon Scheler, leur interprétation «moniste ». A contrario, la « sphère spirituelle » est composée des «personnes» à l'individualité et à la spiritualité irréductibles. Cf. par exemple sur ce point L'homme du ressentiment, trad. fr. M. de Gandillac, Paris, Gallimard, 1933, p. 75, note 1 : «Les actes purement spirituels, leur structure, leur objet, leur unité concrète, ne sauraient être réduits à la "vie", quel que soit le sens philosophique que l'on donne à ce mot..." Dans Nature et formes de la sympathie, Scheler note également, p. 328 : " La compréhension, en tant que compréhension de l'acte et de son sens objectif, n'est autre chose qu'une participation d'un être spirituel à la vie d'un autre être spirituel, participation n'ayant rien à voir avec la perception et indépendante d'elle [...]. Mais la personne, en tant qu'entité spirituelle, outre qu'elle est inobjectivable, est encore trans-intelligible (et en cela elle diffère radicalement de toute existence morte ou interne et de tout ce qui est purement "vital") pour toute connaissance spontanée, car elle est libre de se révéler et de se faire connaître, ou non. » Cette thèse de la connaissance « directe » d'autrui comme « personne » est l'objet du chapitre III de la troisième partie du livre, que Scheler introduit ainsi : "Aussi bien dans notre ouvrage Formalismus in der Ethik que dans 1'“Appendice" à la première édition de l'ouvrage présent, nous n'avons peut-être pas suffisamment insisté sur la différence, pourtant profonde, qui existe entre la manière dont nous sont "donnés" les centres vitaux, psycho-physiques, extérieurs à nous (homme, animal ou plante) et celle dont nous percevons les centres spirituels extérieurs, c'est-à-dire les centres qui font d'un être vivant (de l'homme) une personne... » Il n'est pas inexact de soutenir que le projet de Michel Henry consistera à apporter une autre réponse à ce même problème, en diagnostiquant notamment ce qui, dans la solution de Scheler, subsiste de « continuité » inaperçue entre ce qu'il nomme ces deux « sphères ». Et de fait, 
tout en conservant l'idée d'une ipséité constitutive de l'individu, c'est cet ordre hiérarchique que contestera Henry, comme il le soulignera explicitement, et précisément en référence à Scheler, dans "La vie », conférence prononcée à Poitiers en 1992 à l'occasion du XXIVe Congrès international de l'Association des Sociétés de Philosophie de Langue Française, puis publié dans La Vie et la mort, Poitiers, Société Poitevine de Philosophie, 1996, avant d'être reprise dans Les dossiers H. Michel Henry, Lausanne, L'Âge d'Homme, 2009, p. 91-100 ; cf. notamment p. 95 ; «Lorsqu'on regarde le monde, c'est-à-dire lorsqu'on considère toute chose de l'extérieur, demandant ainsi à l'extériorité l'accès de ces choses et peut-être aussi leur essence, on se trouve en présence d'une hiérarchie qui va de soi : l'inorganique, le vivant, l'homme. Dans cette hiérarchie, la vie occupe une place intermédiaire qui explique sans doute le grand embarras de la pensée à son égard. Même un philosophe immense comme Max Scheler, lorsqu'il entreprendra de renouveler entièrement la problématique de l'intersubjectivité, restera prisonnier de cette hiérarchie et verra son effort grandiose compromis par elle. Depuis toujours cependant dans une telle hiérarchie l'homme est plus qu'un vivant, étant un animal pourvu du Logos. De telle façon que, pour nous les hommes, la vie ne peut se comprendre que difficilement et par une sorte de soustraction par rapport à ce que nous sommes [...]. Seulement, si la vie rend possible l'intentionnalité elle-même et ainsi les actes noétiques que Scheler place au-dessus de la sphère cosmo-vitale, si d'autre part cette vie s'auto-révèle avant qu'un monde soit ouvert et indépendamment de lui, cette hiérarchie qui va de soi instituée depuis toujours entre vie et logos ne doit-elle pas être renversée - la vie n'est-elle pas le logos fondamental, l'Archi-Révélation? » On trouvera un passage presque identique dans " Le corps vivant », Cahiers de l'École des sciences philosophiques et religieuses, $18:$ : Le corps : sujet ou objet? », 1995, repris dans Auto-donation. Entretiens et conférence, Paris, Beauchesne, 2004, p. 129-130.

${ }^{6}$ À trois reprises au moins dans l'œuvre publiée - Philosophie et phénoménologie du corps, op. cit., p. 293, note 1 ; Incarnation. Une philosophie de la chair, Paris, Seuil, 2000, p. 302 ; «Entretien avec Virginie Caruana », dans Entretiens, Arles, Sulliver, 2005, p. 118 - Henry cite la deuxième des Elégies de Duino et son «baiser des amants" pour illustrer l'impossibilité pour l'amour, comme détermination immanente de la vie, de transparaître dans la sphère transcendante du monde - ou, comme il l'écrit ici, dans la "réflexion "; cf. R-M. Rilke, Elégies de Duino, deuxième élégie, dans Euvres poétiques et théâtrales, trad. fr. J-P. Lefebvre, Paris, Gallimard, «Bibliothèque de la Pléiade », p. 532 : "Ainsi vous promettezvous presque / de l'étreinte l'éternité. Et cependant, quand vous surmontez les effrois / des premiers regards et le désir nostalgique à la fenêtre, / et la première promenade ensemble, une seule fois, dans / le jardin : / amoureux, êtes-vous encore aimants ? Lorsque vous vous portez chacun jusqu'à la bouche de l'autre et mariez vos lèvres - quand / vos philtres se touchent : / ô comme étrangement alors le buveur échappe à l'action »- ou bien, pour ce dernier vers et conformément à la traduction utilisée ici par Henry : "Ah! comme le buveur alors de l'acte étrangement s'évade. »

${ }^{7}$ Allusion à la déclaration qui vient clore Une saison en enfer. Henry citera à deux reprises ce passage dans Philosophie et phénoménologie du corps, op. cit., p. 281 et p. 306.

${ }^{8}$ Ce texte haut en couleurs de H. Miller qu'évoque ici Henry se trouve dans Tropique $d u$ cancer, trad. fr. P. Rivert, préface de Henri Fluchère, Paris, Denoël, 1945, p. 163-164. Henry y renverra explicitement dans Philosophie et phénoménologie du corps, op. cit., p. 298.

${ }^{9}$ Sur cette lecture henryenne de Sartre, cf. notre note suivante.

${ }^{10} \mathrm{La}$ théorie husserlienne de 1 ' " apprésentation » se trouve explicitée — pour nous en tenir aux textes que Henry avait lus à l'époque où il rédigeait cette note - au $\S 50$ de la cinquième des Méditations cartésiennes : «L'expérience est un mode de conscience où l'objet est donné "en original" ; en effet, en ayant l'expérience d'autrui nous disons, en général, qu'il est luimême, "en chair et en os" devant nous. D'autre part, ce caractère d'"en chair et en os" ne 
nous empêche pas d'accorder, sans difficultés, que ce n'est pas l'autre "moi" qui nous est donné en original, non pas sa vie, ses phénomènes eux-mêmes, rien de ce qui appartient à son être propre. Car si c'était le cas, si ce qui appartient à l'être propre d'autrui m'était accessible d'une manière directe, ce ne serait qu'un moment de mon être à moi, et, en fin de compte, moi-même et lui-même, nous serions le même. Il en serait de même pour son organisme s'il n'était rien d'autre qu'un "corps" physique (Körper), unité se constituant dans mon expérience réelle et possible et qui appartînt à ma sphère primordiale comme formée exclusivement par ma "sensibilité". Il doit y avoir ici une certaine intentionnalité médiate, partant de la couche profonde du "monde primordial" qui, en tout cas, reste toujours fondamentale. Cette intentionnalité représente une "co-existence" qui n'est jamais et qui ne peut jamais être "en personne" (leiblich). Il s'agit donc d'une espèce d'acte qui rend "coprésent", d'une espèce d'aperception par analogie que nous allons désigner par le terme d'“apprésentation". » (Méditations cartésiennes, trad. fr. E. Lévinas et G. Peiffer, Paris, Vrin, 1994 [1931] p. 174)

${ }_{11}$ Allusion à la dialectique sartrienne de l'amour, qui constitue l'arrière-fond d'un grand nombre de prises de position de Henry dans ces notes. Rappelons que dans L'être et le néant, l'amour est décrit, avec le langage et le masochisme, comme la première attitude fondamentale envers autrui, sur le fondement de la théorie sartrienne d'autrui comme « regard». Les rapports concrets avec autrui, écrit en effet Sartre, «sont commandés tout entiers par mes attitudes vis-à-vis de l'objet que je suis pour autrui. Et comme l'existence d'autrui me révèle l'être que je suis, sans que je puisse ni m'approprier cet être ni même le concevoir » - le pour-soi, comme on sait, n'est pas — « cette existence motivera deux attitudes opposés : autrui me regarde et, comme tel, il détient le secret de mon être, il sait ce que je suis; ainsi, le sens profond de mon être est hors de moi, emprisonné dans une absence ; autrui a barre sur moi. Je puis donc tenter, en tant que je fuis l'en-soi que je suis sans le fonder, de nier cet être qui m'est conféré du dehors ; c'est-à-dire que je puis me retourner sur autrui pour lui conférer à mon tour l'objectité, puisque l'objectité d'autrui est destructrice de mon objectivité pour autrui. Mais, d'autre part, en tant qu'autrui comme liberté est fondement de mon être-en-soi, je puis chercher à récupérer cette liberté et à m'en emparer, sans lui ôter son caractère de liberté : si je pouvais, en effet, m'assimiler cette liberté qui est fondement de mon être en soi, je serais à moi-même mon propre fondement. Transcender la transcendance d'autrui ou, au contraire, l'engloutir en moi cette transcendance sans lui ôter son caractère de transcendance, telles sont les eux attitudes primitives que je prends vis-à-vis d'autrui » (L'être et le néant. Essai d'ontologie phénoménologique, Paris, Gallimard, « Tel», 1991 [1943], p. 403). Certes, contrairement à ce que semble d'abord suggérer Henry ici, c'est la première de ces attitudes, et non la seconde, que, selon Sartre, illustre l'amour : dans l'amour, il ne s'agit justement pas de saisir autrui comme « objet» - ce qui consisterait à supprimer mon être-pour-autrui, et ainsi le phénomène d'autrui en tant que tel — mais de tenter de me saisir, de posséder autrui dans sa liberté. D'où la contradiction d'une telle attitude qui, comme le reconnaît explicitement Sartre - et c'est également à ce titre que Henry la met en question - fait écho à « la fameuse description hégélienne des rapports du maître et de l'esclave» (ibid., p. 410) : l'amant « veut être aimé par une liberté et réclame que cette liberté comme liberté ne soit plus libre» (ibid., p. 407). Si l'être-objet d'autrui n'est donc pas ce que vise l'amour, c'est pourtant ce que l'amour obtient en raison de la contradiction interne de la dialectique amoureuse comme tentative impossible d'objectiver la liberté en tant que liberté : «L'amour n'exige pas l'abolition de la liberté de l'autre, mais son asservissement en tant que liberté, c'est-à-dire son asservissement par elle-même » (Ibid., p. 443). Or c'est justement ce qu'entend contester Henry, en contestant cette dialectique elle-même : parce que l'amour n'est pas désir de possession d'une liberté, il n'échoue pas sur l'être-objet d'autrui, mais se réalise au contraire dans un "contact» avec sa subjectivité en tant que telle. Dans la présente note, Henry fait plus précisément allusion aux descriptions que Sartre donne de la caresse comme désubjecti- 
vation du corps subjectif d'autrui. Chez Sartre toutefois, une telle désubjectivation ne correspond précisément pas à une simple "objectivation», et le corps caressé n'en est pas pour autant assimilé à un pur « objet ». Il s'agit plutôt, dans la caresse, d'une réduction de la corporéité subjective à sa dimension purement facticielle - corps désubjectivé et facticiel donc que Sartre nomme, dans un usage désormais phénoménologiquement équivoque du terme, « chair » : «La caresse révèle la chair en déshabillant le corps de son action, en le scindant des possibilités qui l'entourent : elle est faite pour découvrir sous l'acte la trame d'inertie c'est-à-dire le pur "être-là" — qui le soutient : par exemple en prenant et en caressant la main de l'autre, je découvre, sous la préhension que cette main est d'abord, une étendue de chair et d'os qui peut être prise...» (ibid., p. 430). Or c'est justement à ce corps facticiel - à ce pur " être-là », comme il y reviendra en évoquant H. Miller - que s'oppose, selon Henry, le corps érotique comme corps vivant.

${ }^{12}$ De fait, les questions ici évoquées ne seront qu'esquissées dans la conclusion de Philosophie et phénoménologie du corps, op. cit., p. 302, en note.

${ }^{13}$ Roman de l'écrivain anglais James Hadley Chase, dont la première version parut en 1939, et fut traduite en français en 1946 par M. Duhamel pour la collection « série noire » des éditions Gallimard.

${ }^{14}$ Pour une explicitation de ce concept husserlien, voir la note 10 de la présente édition. Quant à celui d' " analogon », c'est au Sartre de L'imaginaire que Henry l'emprunte ici, et à sa définition de la conscience d'image comme "acte qui vise dans sa corporéité un objet absent ou inexistant à travers un contenu physique ou psychique qui ne se donne pas en propre, mais à titre de représentant analogique de l'objet visé » (Paris, Gallimard, " Folio essai », 1986 [1940], p. 109).

${ }^{15} \mathrm{Cf}$. sur ce point L'être et le néant, op. cit., p. 437.

${ }^{16}$ Pour l'introduction du concept de «possession » dans l'expérience d'autrui, cf. L'être et le néant, op. cit., p. 404 sqq. Mais c'est surtout à la théorie sartrienne du désir et de la chair, sur laquelle nous avons insisté plus haut, que se réfère ici Henry: «Le désir est une tentative pour déshabiller le corps de ses mouvements comme de ses vêtements et de le faire exister comme pure chair ; c'est une tentative d'incarnation du corps d'autrui. C'est en ce sens que les caresses sont appropriation du corps de l'autre... » (ibid., p. 430).

${ }^{17}$ Sur cette question des « deux ordres de fait» chez Maine de Biran - qui annonce bien entendu celle de la «duplicité de l'apparaître » - cf. Philosophie et phénoménologie du corps, op. cit., chapitre I, «Les présupposés philosophiques de l'analyse biranienne du corps », et chapitre IV, «Le double emploi des signes et le problème de la constitution du corps propre $»$.

${ }^{18}$ Allusion à P. Lachièze-Rey, L'idéalisme kantien, Paris, Alcan, 1931, chapitre III a), «La jonction des deux moi », p. 149-207.

${ }^{19} \mathrm{Cf}$. la note 6 de la présente édition.

20 À cette époque, Michel Henry projetait en effet d'écrire un récit intitulé La faim - dans lequel la faim aurait représenté la subjectivité, mais aussi l'amour comme « dimension sacrée de notre vie »; voir sur ce point l'entretien entre A. Henry et J. Leclercq dans Les dossiers $H$. Michel Henry, op. cit., p. 19.

${ }^{21}$ Sur cette interprétation du corps - ou plus précisément de la « chair » — comme détermination « existentielle » dans le christianisme, cf. la conclusion de Philosophie et phénoménologie du corps, op. cit., notamment p. 88 : « Ni le salut, ni le péché ne peuvent [...] être rapportés, en tant que tels, à des structures ontologiques. La "chair" et l'“esprit" désignent l'un comme l'autre, dans le christianisme, des modes spécifiques de l'existence, qui s'opposent sans doute d'une façon radicale quant à la valeur religieuse qui leur est conférée et quant à la signification métaphysique qu'ils reçoivent, par suite, en ce qui concerne le destin de l'homme, mais qui n'en restent pas moins deux modes de l'existence, c'est-à-dire deux inten- 
tionnalités appartenant comme telles à la même sphère ontologique de la subjectivité absolue. »

${ }_{22}$ Allusion à Luc 24:5 : «Pourquoi chercher parmi les morts Celui qui est vivant», s'entendent dire celles qui s'étonnent de la vacuité du tombeau. On trouve une référence analogue à deux endroits dans l'œuvre publiée («Le temps phénoménologique et le présent vivant », dans Autodonation. Entretiens et conférences, Paris, Beauschesne, 2004, p. 59, et «Qu'est-ce que cela que nous appelons la vie ? » dans Phénoménologie de la vie, tome I, De la phénoménologie, Paris, PUF, « Epiméthée », 2003, p. 55), chaque fois dans le but de marquer l'irréalité du " passé transcendant », et en lien avec Le plus proche village de Kafka. Sur ce point, voir Michel Henry, Notes préparatoires à L'essence de la manifestation : la subjectivité, dans Revue Internationale Michel Henry, ${ }^{\circ} 3$, Presses universitaires de Louvain, 2012, et notre présentation, « La subjectivité, la vie, la mort », p. 15-92.

${ }^{23}$ Allusion aux «stades » kierkegaardiens de l'esthétique et du religieux, omniprésents dans les notes préparatoires à Philosophie et phénoménologie du corps et à L'essence de la manifestation.

${ }_{24}$ Probable référence à Philosophie et phénoménologie du corps, dont M. Henry devait, à l'époque où il griffonnait cette note, terminer la rédaction. C'est en tout cas cette question de la contingence qui se trouve en effet posée en conclusion, notamment p. 262 sqq.

${ }^{25}$ Cf. sur ce point L'être et le néant, op. cit., p. 437 : «Le plaisir est la mort et l'échec du désir ». D'une part en ceci qu' "il n'est pas seulement son achèvement mais son terme et sa fin ». Mais d'autre part — et c'est là ce qui retient l'attention de Henry — parce qu'il tend à rompre le charme de l'incarnation: «Le plaisir est l'écluse du désir parce qu'il motive l'apparition d'une conscience réflexive de plaisir, dont l'objet devient la jouissance, c'est-àdire qui est attention à l'incarnation du pour-soi réfléchi et, du même coup, oubli de l'incarnation de l'autre. »

${ }^{26}$ Pour cette critique de Scheler, voir Michel Henry, Textes inédits sur l'expérience d'autrui, op. cit., p. 87 sqq., et le cours d'Aix-en-Provence sur « La communication des consciences et les relations avec autrui », ibid., p. 145 sqq.

${ }^{27}$ Référence à L'être et le néant, op. cit., p. 649 : « L'amour, tel que le décrit Stendhal, apparaît comme un mode d'être dans le monde, c'est-à-dire un rapport fondamental du pour-soi au monde et à soi-même (ipséité) à travers telle femme particulière : la femme ne représente qu'un corps conducteur qui est placé dans le circuit».

${ }^{28}$ Pour cette critique de la dialectique, cf. Michel Henry, Textes inédits sur l'expérience d'autrui, op. cit., p. 91 sqq.

${ }^{29}$ Henry cite une nouvelle fois la deuxième des Elégies de Duino, op. cit., p. 531.

${ }^{30}$ Sur cette lecture de Sartre, voir la note 11 de la présente édition.

${ }^{31} \mathrm{Cf}$. la note 29 de la présente édition.

${ }^{32}$ Sur cette lecture de Sartre, voir la note 11 de la présente édition.

${ }^{33}$ Sur cette critique du caractère unilatéral de la dialectique hégélienne, cf. Michel Henry, Textes inédits sur l'expérience d'autrui, op. cit., p. 91 sqq., et notre présentation, «De l'expérience métaphysique d'autrui à l'intersubjectivité en première personne ", op. cit., p. $42 s q q$.

${ }^{34}$ Référence à A. Koyré, La philosophie de Jacob Boehme, Paris, Vrin, 1929, p. 158, ouvrage qui, si l'on en croit les notes préparatoires à L'essence de la manifestation, avait beaucoup impressionné Henry, et auquel renverra par deux fois le texte publié (Paris, PUF, «Epiméthée », 1990, p. 139 et p. 148).

${ }^{35}$ Sur cette opposition du christianisme à la dialectique pour la reconnaissance, cf. Michel Henry, Textes inédits sur l'expérience d'autrui, op. cit., et notamment le Ms C 9-471-2966: «Expérience d'autrui. Lorsque le Christ dit aimez-vous, que fait-il d'autre que trancher d'un coup de sabre les nœuds sans fin de la dialectique du Maître et de l'Esclave ? Que couper le 
ressentiment (d'où absurdité de la critique de Nietzsche ; ainsi la faiblesse du christianisme est en réalité sa suprême lucidité). Que comprendre qu'être le maître d'autrui (se venger, etc.) c'est aboutir à l'échec de tous les maîtres : à la solitude métaphysique et à la ruine. »

${ }^{36} \mathrm{Cf}$. sur ce point la note 8 de la présente édition.

${ }^{37}$ Allusion à D. Lagache, La jalousie amoureuse, Paris, PUF, 1947, auquel Henry se réfère à différentes reprises dans les notes préparatoires à Philosophie et phénoménologie du corps et à L'essence de la manifestation.

${ }^{38}$ Sur cette question de la profanation, cf. Michel Henry, Incarnation, op. cit., § 43 (« La réduction de la relation érotique à la sexualité objective au temps du nihilisme »), notamment p. 313-315.

39 Sur le «paradoxe kierkegaardien » comme synthèse de l' «âme » et du « corps », cf. Incarnation, op. cit., §38 («La duplicité de l'apparaître et le redoublement de l'angoisse ») ; pour un traitement thématique de la question de l'innocence, cf. Incarnation, § 37 («L'oubli de la vie et son rappel pathétique dans l'angoisse ») ; Sur le «paradoxe kierkegaardien» comme synthèse de l' «âme » et du « corps », cf. ibid., § 38 («La duplicité de l'apparaitre et le redoublement de l'angoisse »).

${ }^{40}$ Sur le thème du menteur dans son lien avec la question de la « simulation », cf. la conclusion de C'est moi ma vérité. Pour une philosophie du christianisme, Paris, Seuil, 1996, notamment p. 336-345. Pour une articulation précise de ces "catégories" de l'érotisme, cf. Incarnation, op. cit., § 43, p. 313 sqq.

${ }^{41}$ Sur cette question, ici centrale, de l'auto-érotisme, voir notamment $C$ 'est moi la vérité, op. cit., p. 258 sqq., et Incarnation, op. cit., § 32 («Retour à la thèse de Condillac. L'autoérotisme de la statue : la chair comme lieu de la perdition. Passage nécessaire d'une phénoménologie de la chair à une phénoménologie de l'Incarnation») et $\S 41$ («La relation érotique dans l'immanence de la vie : l'échec du désir »).

${ }^{42}$ Sur cette question de la nudité et du dénudement, cf. Incarnation, op. cit., § 43, notamment p. 311-315.

${ }^{43}$ Sur cette question de l'idolâtrie, cf. Incarnation, op. cit., §§ 45-46; pour le lien entre profanation, idolâtrie et «illusion transcendantale de l'ego », cf. notamment p. 326 sqq.

${ }^{44}$ Référence à Irénée de Lyon, Contre les hérésies, Paris, Le Cerf, 1991, p. 366. Cette page se trouve commentée dans Incarnation, op. cit., § 46 (« La voie du salut selon Irénée et selon Augustin »), p. 335 sqq.

${ }^{45}$ Sur ce thème de "l'illusion transcendantale de l'ego », cf. C'est moi la vérité, op. cit., chap. 8 : "L'oubli par l'homme de sa condition de Fils : "Moi, je" ; "Moi, ego" ", notamment p. 176-179 : «Éprouvant chacun de ses pouvoirs, tandis qu'il l'exerce, et d'abord le pouvoir qu'il a de les exercer, l'ego se prend [...] pour leur source, pour leur origine. Il s'imagine qu'il possède ces pouvoirs, qu'ils sont les siens en un sens radical - comme ce qu'il aurait produit lui-même et aussi bien comme ce qu'il produirait à chaque instant tandis qu'il les exerce. Source et origine en quelque sorte absolues des pouvoirs qui composent son être l'être effectif et agissant avec lequel il s'identifie et par lequel il se définit —, il se considère aussi bien comme la source et l'origine de son être même. Ainsi naît l'illusion transcendantale de l'ego, illusion par laquelle cet ego se prend pour le fondement de son être. [...] [Mais] L'illusion transcendantale de l'ego n'est pas totalement illusoire [...]. Elle comporte une part de "réalité" et de "vérité" dont il faut prendre la mesure, parce qu'elle est tout simplement essentielle. Le don par lequel la Vie se donnant à soi donne l'ego à lui-même, ce don en est un. Donné à soi, l'ego est réellement en possession de lui-même et de chacun de ses pouvoirs, en mesure de les exercer : il est réellement libre. En faisant de lui un vivant, la Vie n'en a pas fait un pseudo-vivant. Elle ne reprend pas d'une main ce qu'elle a donné de l'autre. [...] Je Peux - la mise en œuvre effective de chacun de mes pouvoirs - est le contraire d'une illusion. De même en est-il pour le "Je suis" qui naît de ce "Je Peux". Ainsi l'effectivité de ce "Je 
Peux"/ "Je suis" vient-elle recouvrir le fait que ce "je peux" vivant, ce "je suis" vivant, n'advient que par l'œuvre, qui ne cesse pas, de la Vie en lui.» Cette thématique se trouvera reprise dans Incarnation, op. cit., §35 («Illusion et réalité du "je peux" »), et son $\S 41$ (« La relation érotique dans l'immanence de la vie : l'échec du désir ») en développera les implications concernant la problématique de l'érotisme.

${ }^{46}$ Sur ce thème du « vertige » et du « vertigineux », caractéristique de la lecture henryenne de Kierkegaard en tant qu'elle lie intrinsèquement la question de l'érotisme à celle de la liberté comme «possibilité de pouvoir», cf. Incarnation, op. cit., § 37 («L'oubli de la vie et son rappel pathétique dans l'angoisse »); pour la critique du «Sehen und Fassen», cf. ibid., p. 105 et p. 162

${ }^{47}$ Cf. Romains 6, 1-18 : «Que dirons-nous donc ? Demeurerons-nous dans le péché, afin que la grâce abonde? Loin de là ! Nous qui sommes morts au péché, comment vivrons-nous encore dans le péché ? Ne savez-vous pas que nous tous qui avons été baptisés en JésusChrist, c'est en sa mort que nous avons été baptisés ? Nous avons donc été ensevelis avec lui par le baptême en sa mort, afin que, comme le Christ est ressuscité des morts par la gloire du Père, nous aussi nous marchions dans une vie nouvelle. Si, en effet, nous avons été greffés sur lui, par la ressemblance de sa mort, nous le serons aussi par celle de sa résurrection : sachant que notre vieil homme a été crucifié avec lui, afin que le corps du péché fût détruit, pour que nous ne soyons plus les esclaves du péché ; car celui qui est mort est affranchi du péché. Mais si nous sommes morts avec le Christ, nous croyons que nous vivrons avec lui, sachant que le Christ ressuscité des morts ne meurt plus ; la mort n'a plus sur lui d'empire. Car sa mort fut une mort au péché une fois pour toutes, et sa vie est une vie pour Dieu. Ainsi vous-mêmes regardez-vous comme morts au péché, et comme vivants pour Dieu en Jésus-Christ [NotreSeigneur]. Que le péché ne règne donc point dans votre corps mortel, de sorte que vous obéissiez à ses convoitises. Ne livrez pas vos membres au péché pour être des instruments d'iniquité, mais offrez-vous vous-mêmes à Dieu comme étant vivants, de morts que vous étiez, et offrez-lui vos membres pour être des instruments de justice. Car le péché n'aura pas d'empire sur vous, parce que vous n'êtes pas sous la Loi, mais sous la grâce. Quoi donc! Pécheronsnous, parce que nous ne sommes pas sous la Loi mais sous la grâce ? Loin de là ! Ne savezvous pas que, si vous vous livrez à quelqu'un comme esclaves pour lui obéir, vous êtes esclaves de celui à qui vous obéissez, soit du péché pour la mort, soit de l'obéissance à Dieu pour la justice? Mais grâces soient rendues à Dieu de ce que, après avoir été les esclaves du péché, vous avez obéi de cœur à la règle de doctrine qui vous a été enseignée. Ainsi, ayant été affranchis du péché, vous êtes devenus les esclaves de la justice. » 

VA R I A 



\section{VARIA}

Première section

Philosophie de la religion 
